\title{
Three-factor Fuzzy Information Channel Model Representing Subjective Judgments Using Entropy that Integrates both Randomness and Fuzziness
}

\author{
Mamoru UEHARA ${ }^{\mathrm{a}^{*}}$, Masanobu MATSUMARU ${ }^{\mathrm{b}}$ \\ ${ }^{a}$ Department of Business, Faculty of Business, Aichi-Shukutoku University, \\ 23 Sakuragaoka, Chikusa-ku, Nagoya, Aichi 464-8671, Japan \\ ${ }^{b}$ Research Institute for Engineering, Kanagawa University, \\ 3-27-1 Rokkakubashi, Kanagawa-ku, Yokohama, Kanagawa, 221-8686 Japan
}

\begin{abstract}
Numerous multicriteria decision-making (MCDM) methods have been proposed to support decision making: the evaluation and selection of alternatives based on multiple criteria. This study proposes a new three-factor fuzzy information channel model by expanding a two-factor fuzzy information channel model for MCDM problems. The two-factor fuzzy information channel model estimates criteria selection ratios for two selection criteria: one quantitative crisp criterion and one qualitative fuzzy criterion. We propose a three-factor fuzzy information channel model (model 1, weightless) to handle three criteria by increasing the number of qualitative fuzzy criteria to two. Next, we propose a weighted fuzzy information channel model (model 2, weighted) that considers the subjective weights of decision makers (DMs) for criteria. The proposed models resolve MCDM problems based on randomness-fuzziness entropy using both entropy for randomness and entropy for fuzziness, and can estimate the selection ratios of criteria based on a simple model. We study the performance of the proposed models by comparing them with the fuzzy multiobjective linear model, which is another MCDM method, using a practical supplier selection example.
\end{abstract}

Keywords: Multicriteria decision making; Fuzzy multicriteria decision making; Randomness-fuzziness entropy; Fuzzy information channel model; Human information processing; Supplier selection

\section{Introduction}

Researchers and practitioners have widely studied multicriteria decision making (MCDM) to support decision making to evaluate and select alternatives based on multiple criteria. Zavadskas and Turskis [1], Liou and Tzeng [2], and Zavadskas et al. [3] reviewed the most advanced technologies reported in the literature, and they introduced and organized analysis methods related to MCDM. Real-world decision-making problems are based not only on clear quantitative information, but also on qualitative information characterized by uncertainty and vagueness. Many fuzzy multicriteria decisionmaking (FMCDM) methods applying fuzzy sets have been proposed to overcome this problem. FMCDM is a decision-making methodology widely used in engineering, technology, science, management, and business. Kahraman et al. [4] surveyed and categorized state-of-the-art FMCDM methods, while HerreraViedma [5] discussed the effectiveness of fuzzy technologies in MCDM. Mardani et al. [6] reviewed 403 papers on FMCDM published from 1994 to 2014 and categorized them mainly into four groups: engineering, management and business, science, and technology. Furthermore, they classified the papers by date of publication, method, tool, and type of research.

The fact that probabilistic uncertainty decreases through the acquisition of information is reflected in

*Corresponding Author: muehara@asu.aasa.ac.jp

Received: December 15, 2018

Accepted: November 8, 2019
Shannon's information theory of systematization based on probability theory, in which entropy plays a fundamental role [7]. The one-factor information channel model that used Shannon's entropy [8] was originally built to explain the selection behavior of the public as a social phenomenon. Subsequently, it was widely used as a model to describe many social phenomena in a comparatively simple form.

Since Zadeh's work [9], there have been many attempts to ascertain the vagueness with regard to meaning in human information processing by using fuzzy theory. On the one hand, in a crisp set in conventional set theory, it must be possible to clearly distinguish whether individual elements belong to a set. On the other hand, in fuzzy theory, the boundaries of a fuzzy set may be unclear and vague.

De Luca and Termini [10] proposed fuzzy entropy to measure the degree of fuzziness of an overall set when the vagueness of the object being targeted is expressed with a fuzzy set. It is known that fuzzy entropy is appropriate as an effective measure of fuzziness [11] Accordingly, fuzzy entropy is widely applied as an indicator, and the search for an information concept that ascertains the type of uncertainty from a different perspective in this area has been described as extremely important for the further development of information science [7]. Therefore, many FMCDM methods using 
the fuzzy entropy of De Luca and Termini [10] have been proposed [12-17].

Yamashita [18] and Nishikawa et al. [19] focused on Shannon's entropy based on probability theory and on the fuzzy entropy of De Luca and Termini [10] to propose a randomness-fuzziness entropy that expresses the dual nature of vagueness in information processing methods of individuals and organizations. The dual nature here signifies two aspects: vagueness surrounding the question "which will you choose?" (vagueness relating to randomness) and vagueness surrounding the question "what meaning does it have?" (vagueness relating to fuzziness). Randomness-fuzziness entropy ascertains the former through probability, as in the entropy model, and the latter through the membership functions of fuzzy sets. Yamashita [20] treats randomness-fuzziness entropy as an index representing vagueness that integrates both randomness and vagueness. In addition, to expand the one-factor information channel model, which is an entropy model, to a fuzzy entropy model, a one-factor fuzzy information channel model was proposed by introducing randomness-fuzziness entropy to a one-factor information channel model.

Factors of the one-factor fuzzy information channel model are the objects of vague information. However, in practical cases, decision makers (DMs) often have to make difficult decisions based on both clear quantitative information and qualitative, uncertain, and insufficient information. Therefore, Yamashita [21] proposed a twofactor fuzzy information channel model by expanding the one-factor fuzzy information channel model to handle two factors, involving clear quantitative information and vague information that is qualitative and uncertain. Even if information necessary for judgments is verbalized or formalized in rules, uncertain information that cannot be fully formalized will inevitably be scattered in formalized rules and languages. A benefit of performing MCDM based on both vagueness concerning fuzziness and vagueness concerning randomness in this manner is that it uses randomness-fuzziness entropy.

Several review papers have recently reported on MCDM [1, 2] and FMCDM [6] methods. However, to our knowledge, no research has been reported on the evaluation and selection methods using randomnessfuzziness entropy applied by extending fuzzy entropy. The present study aims to expand the application range of selection criteria by handling one quantitative crisp criterion and two qualitative fuzzy criteria using randomness-fuzziness entropy. We achieved this objective by proposing a three-factor fuzzy information channel model obtained by expanding the two-factor fuzzy information channel model of Yamashita [21]. In practical cases, DMs make decisions by attaching various weights to the selection criteria according to strategic and management policies. Accordingly, this proposed model is expanded to a weighted three-factor fuzzy information channel model that reflects the DM's evaluation weights for the criteria. Therefore, we propose a decision-making model that decides the selection ratios by considering the weight of the DM.

The features and advantages of the models presented herein are as follows:

(a) The models proposed in this study can express a DM by considering both quantitative information and qualitative, fuzzy information.

(b) The weighted model proposed in this study considers the DM's subjective weights for criteria (in the model, subjective weights are expressed as a coefficient).

(c) Recent MCDM methods are shifting from methods based on independent criteria in a hierarchical structure (e.g., AHP) to interdependent methods, which do not assume a hierarchical structure. In our proposed models, for a myriad of criteria other than those considered in our models, we can consider not only independent criteria in a hierarchical structure but also the influence of interdependent criteria, which do not assume a hierarchical structure. This realizes the formulation of a very simple model of entropy or fuzzy entropy maximization.

(d) Our models are simple and can be easily solved (with spreadsheet software).

The supplier evaluation and selection problem has also recently been extensively studied. However, when selecting a supplier, the DMs must use clear quantitative and qualitative information in uncertain forms and with insufficient supporting evidence to evaluate suppliers, which makes these decisions difficult. In addition, many criteria must be considered when selecting the optimal supplier after understanding the supplier's performance. Therefore, DMs who select suppliers must evaluate and select suppliers considering multiple criteria, which makes the process an MCDM problem. The three-factor fuzzy information channel model and weighted threefactor fuzzy information channel model presented here that use randomness-fuzziness entropy are useful for solving this problem. We evaluated the effectiveness of our proposed models in comparison with other FMCDM methods for the supplier selection problem by using numerical examples.

The remainder of this paper is structured as follows: Some basic theories required for the proposed models are introduced in Section 2; the assumptions and motivations for this research are explained in Section 3; our methods and algorithms are presented in Section 4. A comparative study of our proposed methods and other methods, taking the problem of supplier selection as a sample application, is discussed in Section 5; finally, we present our conclusions and further research recommendations in Section 6.

\section{Preliminaries}

\subsection{Multiple-criteria decision making}

MCDM is considered a complex decision-making tool that evaluates and selects alternatives based on multiple criteria involving both quantitative and qualitative factors [6]. In recent years, several FMCDM tools have 
been suggested for choosing optimal options [6]. Liou and Tzeng [2] conducted the latest literature survey of MCDM methods. They organized the MCDM methods in an easy-to-understand manner as follows: First, they roughly separated MCDM into multiobjective decisionmaking (MODM) and multiattribute decision-making (MADM) components. Furthermore, they categorized MADM into three classes: (1) structure relation methods, also called evaluating or choosing models (e.g., decisionmaking trial and evaluation laboratory (DEMATEL), fuzzy DEMATEL, interpretive structural modeling (ISM), fuzzy ISM, fuzzy cognitive map (FCM), linear structure equation model (LISEM or SEM), formal concept analysis, and input-output analysis); (2) weight analysis, also referred to as weighting models (e.g., analytic hierarchy process (AHP)/fuzzy AHP, analytic network process (ANP)/fuzzy ANP, entropy measure, neural network weighting, or dynamic weighting); and (3) performance-aggregated methods, also called normalizing models (additive types: technique for order preference by similarity to an ideal solution (TOPSIS), simple additive weight (SAW), VIKOR, ELECTRE, and gray relation; nonadditive types: fuzzy integral).

Four studies using fuzzy entropy were discussed in the literature survey by Mardani et al. [6], but these studies use fuzzy entropy as a weighting model. In recent years, more sophisticated and hybrid advanced MCDM and FMCDM techniques have been developed by many researchers. However, no studies used randomnessfuzziness entropy, which is an extension of fuzzy entropy used in the present study for evaluating or choosing models.

\subsection{One-factor information channel model}

We introduce herein Kunisawa's [8] one-factor information channel model. This model treats the problem of estimating the selection ratio $p_{i}$ by applying the characteristic value $x_{i}$ for alternative $i$, which is the object of selection behavior. In the one-factor information channel model, this type of consumer behavior is formulated from both the "levels of satisfaction regarding the attributes that give each product and service their characteristics (price, fees, number of sales, etc.)" and "free and selfish selection behavior."

In the one-factor information channel model [8], the following two hypotheses were established for these two aspects, which are ascertained by the average characteristic value $L$ in Equation (1) for Hypothesis I and entropy $E$ in Equation (2) for Hypothesis II.

Hypothesis I: When consumers select any product or service, they try to spend as little as possible of their own money (generally, they try to make the characteristic value $x_{i}$ for a certain alternative $i$ as small as possible).

Hypothesis II: When consumers select any product or service, they try to select as freely and as selfishly as possible through their own decisions without any kind of constraint.

$$
\begin{aligned}
& L=\sum_{i=1}^{n} p_{i} \cdot x_{i} \\
& E=-\sum_{i=1}^{n} p_{i} \cdot \log p_{i}
\end{aligned}
$$

where

$i$ : alternative

$n$ : number of samples

$p_{i}$ : selection ratio (the sum of $p_{i}$ values is considered to be 1)

$x_{i}$ : characteristic value

In accordance with hypotheses I and II, the selection ratio $p_{i}$ is estimated, such that it is formulated using the Lagrange undefined multiplier $\lambda$ as an entropy maximization problem of $R$ in Equation (3) [8]. The selection ratio $p_{i}$ can be estimated by solving Equation (3). The $p_{i}$ calculation method is described in Appendix A.

$$
R=\frac{E}{L}-\lambda \cdot\left(\sum_{i=1}^{n} p_{i}-1\right) \rightarrow \max .
$$

\subsection{Fuzzy entropy}

In this section, we introduce the fuzzy entropy reported by De Luca and Termini [10]. When considering the function for a measure of fuzziness, $d$, and the set family for $X$ as $F(X)$ (this element is fuzzy set $A$ ), $d$ is mapped to $[0,+\infty]$ from $F(X)$. Practically, many $d(A)$ exist, but as a fundamental functional type, De Luca and Termini [10] defined it as follows:

$$
\begin{aligned}
d(A) & =k \sum_{i=1}^{n}\left[-\mu_{A}\left(x_{i}\right) \cdot \log \mu_{A}\left(x_{i}\right)\right. \\
& \left.-\left(1-\mu_{A}\left(x_{i}\right) \cdot \log \left\{\left(1-\mu_{A}\left(x_{i}\right)\right)\right\}\right)\right],
\end{aligned}
$$

where $k$ is a constant, such that $0 \leqq d(A) \leqq 1 . \mu_{A}\left(x_{i}\right)$ are the fuzzy values. De Luca and Termini [10] termed this function fuzzy entropy.

\subsection{Randomness-fuzziness entropy}

As a method of ascertaining the aspects of both fuzziness and randomness that constitute the dual nature of vagueness, Nishikawa et al. [19] positioned the vagueness of output information that combines the randomness of selection probability $q_{j}$ and fuzziness of the degree-of-membership function $\mu_{j}$ for alternative $j$ as "behavior entropy." They then established the following formula from randomness-fuzziness entropy $\left(E_{R F}\right)$ : 


$$
\begin{aligned}
E_{R F}= & \frac{1}{n} \sum_{j=1}^{n}\left[-q_{j} \cdot \mu_{j} \cdot \log q_{j} \cdot \mu_{j}\right. \\
& \left.-q_{j} \cdot\left(1-\mu_{j}\right) \cdot \log \left\{q_{j}\left(1-\mu_{j}\right)\right\}\right],
\end{aligned}
$$

where:

$$
\begin{aligned}
& j: \text { alternative } \\
& n: \text { number of samples } \\
& \mu_{j}: \text { membership value } \\
& q_{j}: \text { selection probability }
\end{aligned}
$$

When considering an identical sample, $1 / n$ is a constant and can therefore be removed. Equation (6) results in the following form when $q_{j}$ and $\mu_{j}$ are arranged:

$E_{R F}=-\sum_{j=1}^{n} q_{j} \cdot \log q_{j}+\sum_{j=1}^{n} q_{j} \cdot H_{j}$

where

$$
H_{j}=-\mu_{j} \cdot \log \mu_{j}-\left(1-\mu_{j}\right) \log \left(1-\mu_{j}\right) .
$$

The first term on the right-hand side of Equation (6) expresses entropy regarding randomness and the second term expresses entropy regarding fuzziness. Entropy regarding randomness corresponds to entropy in Shannon's information theory. Therefore, it represents the average of the quantity of information obtained when it is known which sample was selected [20]. Moreover, entropy regarding fuzziness is the result of weighting entropy $H_{j}$ using alternative $j$ for the question "does alternative $j$ belong or not belong to the fuzzy set?" by selection probability $q_{j}$; it is possible to interpret this as the average of entropy regarding fuzziness.

\subsection{One-factor fuzzy information channel model}

Here, we introduce the one-factor fuzzy information channel model proposed by Yamashita [20]. The factors that are targeted by the one-factor information channel model are clear characteristic values (numerical values), typically with $\operatorname{cost} t_{j}$, but Yamashita expanded this model to include vague characteristic values. Therefore, such vague characteristic values are determined based on membership values in the fuzzy set.

This means that the average characteristic value $L$ of Equation (1) and entropy $E$ of Equation (2) in the onefactor information channel model are converted into Equation (7) (average characteristic value: probability $L_{F 1}$ of the fuzzy phenomenon) and Equation (8) $\left(E_{R F 1}\right)$, which is the same as Equation (6) (randomnessfuzziness entropy: $E_{R F}$ ), respectively:

$L_{F 1}=\sum_{j=1}^{n} q_{j} \cdot \mu_{j}$ where

$$
\begin{aligned}
\sum_{j=1}^{n} q_{j} & =1 \\
E_{R F 1} & =E_{R F} \\
& =-\sum_{j=1}^{n} q_{j} \cdot \log q_{j}+\sum_{j=1}^{n} q_{j} \cdot H_{j}
\end{aligned}
$$

where

$H_{j}=-\mu_{j} \cdot \log \mu_{j}-\left(1-\mu_{j}\right) \log \left(1-\mu_{j}\right)$.

Here, Yamashita [20] formulated Equation (9) using the Lagrange undefined multiplier $\lambda$ to estimate the selection probability $q_{j}$.

$R_{1}=\frac{E_{R F 1}}{L_{F 1}}-\lambda \cdot\left(\sum_{j=1}^{n} q_{j}-1\right) \rightarrow \max$.

As Equation (9) is convex with respect to $\boldsymbol{q}_{\boldsymbol{j}}$, the selection probability $\boldsymbol{q}_{\boldsymbol{j}}$ can be estimated by partially differentiating Equation (9) with respect to $\boldsymbol{q}_{\boldsymbol{j}}$ and setting it to 0 . The detailed calculation method for estimating $\boldsymbol{q}_{\boldsymbol{j}}$ is provided in Appendix B.

\subsection{Two-factor fuzzy information channel model}

In practical cases, DMs often have to make decisions based on both clear quantitative information and qualitative, uncertain, insufficient information. Therefore, Yamashita [21] proposed a two-factor fuzzy information channel model by expanding the one-factor fuzzy information channel model to the problem of handling two factors: not only crisp, quantitative characteristic values (information), but also qualitative, vague characteristic values (fuzzy information). However, this model makes the following assumptions: Crisp and quantitative characteristic values are preferable values when the numerical values are lower, and quantitative and vague characteristic values are preferable when membership values are higher.

Here,

\section{$\boldsymbol{j}$ : alternative}

$\boldsymbol{q}_{\boldsymbol{j}}$ : selection probability (the sum of $\boldsymbol{q}_{\boldsymbol{j}}$ values is considered to be 1)

$\boldsymbol{t}_{j}$ : cost

$\boldsymbol{\mu}_{\boldsymbol{A}}\left(\boldsymbol{P}_{\boldsymbol{j}}\right)$ : the membership degree to which product $\boldsymbol{P}_{\boldsymbol{j}}$ belongs to set $\mathrm{A}$ of high-quality products.

Hence, the average characteristic value $\left(\boldsymbol{L}_{\boldsymbol{F} 2}\right)$ and randomness-fuzziness entropy $\left(\boldsymbol{H}_{\boldsymbol{R F} 2}\right)$ in the two-factor information channel model are formulated using Equations (10) and (11), respectively. 


$$
\begin{aligned}
& L_{F 2}=\sum_{j=1}^{n}\left(\frac{t_{j}}{\mu_{A}\left(P_{j}\right)}\right) \cdot q_{j}, \\
& H_{R F 2}=-\sum_{j=1}^{n} q_{j} \cdot \log q_{j}+\sum_{j=1}^{n} q_{j} \cdot G_{j},
\end{aligned}
$$

where $\boldsymbol{G}_{\boldsymbol{j}}$ is the entropy regarding fuzziness and is defined by Equation (12):

$$
\begin{aligned}
G_{j}= & -\mu_{A}\left(P_{j}\right) \cdot \log \mu_{A}\left(P_{j}\right)- \\
& \left(1-\mu_{A}\left(P_{j}\right)\right) \cdot \log \left(1-\mu_{A}\left(P_{j}\right)\right) .
\end{aligned}
$$

Here, it is formulated using the Lagrange undefined multiplier $\boldsymbol{\lambda}$ as the maximization problem of $\boldsymbol{R}_{\mathbf{2}}$ in Equation (13).

$$
R_{2}=\frac{H_{R F 2}}{L_{F 2}}-\lambda \cdot\left(\sum_{j=1}^{n} q_{j}-1\right) \rightarrow \max
$$

As Equation (13) is convex with respect to $\boldsymbol{q}_{j}$, selection probability $\boldsymbol{q}_{\boldsymbol{j}}$ can be estimated by partially differentiating Equation (13) with respect to $\boldsymbol{q}_{\boldsymbol{j}}$ and setting the differential to 0 . The detailed calculation method for estimating $\boldsymbol{q}_{j}$ is provided in Appendix C.

\section{Assumptions and motivations of the proposed method}

In this method, two assumptions were made when constructing the model. Among the criteria used by DMs to evaluate alternatives are quantitative crisp criteria and qualitative fuzzy criteria. The purpose of this study was to estimate the selection ratio $r_{m}$ of alternative $m$ in the case where both are selection criteria. The first assumption of this study is that the selection ratio $r_{m}$ used here is obtained based on the selection probability in the one-factor fuzzy information channel model and the two-factor fuzzy information channel model reported in previous research. The second assumption of this study is that three types of selection criteria exist: one quantitatively crisp criterion and two qualitatively fuzzy criteria. The quantitatively crisp criterion is assumed to be a preferable criterion as the numerical value is lower, and the qualitatively fuzzy criteria are assumed to be preferable criteria as membership values are higher.

The characteristics of this method include the following four points: First, the problem encountered by DMs when selecting alternatives, which is the fact that the selection criteria are a mixture of one quantitative crisp criterion and two qualitative fuzzy criteria, is resolved by using the randomness-fuzziness entropy, which involves the entropy regarding randomness and entropy regarding fuzziness. Second, to expand the range of application of selection criteria by handling one quantitative crisp criterion and two qualitative fuzzy criteria, we propose a three-factor fuzzy information channel model that expands the two-factor fuzzy information channel model of Yamashita [21] as model 1 (weightless). In practical cases, DMs attach a variety of weights to objectives (or criteria) to make decisions. Therefore, third, we propose a weighted three-factor fuzzy information model as model 2 (weighted) by reflecting the weight of the DMs in model 1. Finally, these models can replicate the human decision-making process in a simple form. Furthermore, these models can easily estimate the selection ratio.

\section{Proposed three-factor fuzzy information channel model}

The three-factor fuzzy information channel model that expands Yamashita's two-factor fuzzy information channel model [21] to three factors (model 1, weightless) and the three-factor fuzzy information channel model that considers the weighting of criteria by DMs (model 2, weighted) are constructed here.

[Model 1] (weightless) Three-factor fuzzy information channel model

In decision making with model 1 , when the degree of membership in the set in selection $\mathbf{z}_{\mathbf{m}}$ (membership value $\left.\boldsymbol{\mu}_{\boldsymbol{A}}\left(\mathbf{z}_{\boldsymbol{m}}\right)\right)$ and the degree of membership in set $\mathrm{B}$ (membership value $\boldsymbol{\mu}_{B}\left(\boldsymbol{z}_{\boldsymbol{m}}\right)$ ) are given; based on Bellman and Zadeh [22], the fuzzy decision $D$ is defined as a fuzzy set $\boldsymbol{A} \cap \boldsymbol{B}$. In other words, for all $\boldsymbol{z}_{\boldsymbol{m}}$,

$\mu_{A \cap B}\left(z_{m}\right)=\min \left[\mu_{A}\left(z_{m}\right), \mu_{B}\left(z_{m}\right)\right]$.

Here, the alternative is $\boldsymbol{m}$, and the selection ratio is $\boldsymbol{r}_{\boldsymbol{m}}$ (the sum of all $\boldsymbol{r}_{\boldsymbol{m}}$ is considered to be 1).

The one quantitative, crisp characteristic value is represented as $\boldsymbol{c}_{\boldsymbol{m}}$; for the two qualitative fuzzy characteristic values, the degree of membership in set $A$ is represented as membership value $\boldsymbol{\mu}_{\boldsymbol{A}}\left(\boldsymbol{z}_{\boldsymbol{m}}\right)$ and the degree of membership in set $B$ as membership value $\boldsymbol{\mu}_{B}\left(\mathbf{z}_{\mathrm{m}}\right)$.

Hence, the average characteristic value $\left(\boldsymbol{L}_{F 3}\right)$ and randomness-fuzziness entropy $\left(\boldsymbol{H}_{\boldsymbol{R} F 3}\right)$ in the three-factor information channel model are formulated using Equations (15) and (16), respectively.

$$
\begin{gathered}
L_{F 3}=\sum_{m=1}^{n}\left(\frac{c_{m}}{\min \left[\mu_{A}\left(z_{m}\right), \mu_{B}\left(z_{m}\right)\right]}\right) \cdot r_{m} \\
H_{R F 3}=-\sum_{m=1}^{n} r_{m} \cdot \log r_{m} \\
+\sum_{m=1}^{n} r_{m} \cdot G_{1 m}
\end{gathered}
$$

where $\boldsymbol{G}_{\mathbf{1 m}}$ is entropy regarding fuzziness and is defined by Equation (17): 


$$
\begin{aligned}
G_{1 m}= & -\min \left[\mu_{A}\left(z_{m}\right), \mu_{B}\left(z_{m}\right)\right] \\
& \cdot \log \left\{\min \left[\mu_{A}\left(z_{m}\right), \mu_{B}\left(z_{m}\right)\right]\right\} \\
& -\left(1-\min \left[\mu_{A}\left(z_{m}\right), \mu_{B}\left(z_{m}\right)\right]\right) \\
& \cdot \log \left\{1-\min \left[\mu_{A}\left(z_{m}\right), \mu_{B}\left(z_{m}\right)\right]\right\} .
\end{aligned}
$$

Here, it is formulated using the Lagrange undefined multiplier $\boldsymbol{\lambda}$ as the maximization problem of $\boldsymbol{R}_{\mathbf{3}}$ in Equation (18).

$$
\begin{aligned}
R_{3}=\frac{H_{R F 3}}{L_{F 3}}-\lambda & \lambda \cdot\left(\sum_{m=1}^{n} r_{m}-1\right) \\
\rightarrow \max . &
\end{aligned}
$$

As Equation (18) is convex with respect to $\boldsymbol{r}_{\boldsymbol{m}}$, partially differentiating Equation (18) with respect to $\boldsymbol{r}_{\boldsymbol{m}}$ and setting the differential to 0 yields the resolution according to the following equation for model 1 :

$$
\begin{aligned}
\boldsymbol{r}_{m} & =\exp \left[\left(\frac{G_{1 m}-c_{m}}{\min \left[\mu_{A}\left(z_{m}\right), \mu_{B}\left(z_{m}\right)\right]}\right)\right] \cdot \frac{H_{R F 3}}{L_{F 3}} \\
& =\exp \left[G_{1 m}\right] \cdot W_{1}^{\frac{c_{m}}{\min \left[\mu_{A}\left(z_{m}\right), \mu_{B}\left(z_{m}\right)\right]}}
\end{aligned}
$$

where $\boldsymbol{W}_{1}=\exp \left[-\frac{H_{R F 3}}{L_{F 3}}\right]$. As the sum of all $\boldsymbol{r}_{\boldsymbol{m}}$ values is $1, \boldsymbol{W}_{\mathbf{1}}$ satisfies Equation (20):

$$
\sum_{m=1}^{n} \exp \left[G_{1 m}\right] \cdot W_{1}^{\frac{c_{m}}{\min \left[\mu_{A}\left(z_{m}\right), \mu_{B}\left(z_{m}\right)\right]}}=1
$$

[Model 2] (weighted) Weighted three-factor fuzzy information channel model

In model 2, the weights of the DMs on the two fuzzy characteristic values $\mu_{A}\left(z_{m}\right)$ and $\mu_{B}\left(z_{m}\right)$ and the one quantitative, crisp characteristic value $c_{m}$ are $w_{A}$, $w_{B}$, and $w_{C}$, respectively. The membership values $\mu_{A}\left(x_{i}\right)$ and $\mu_{B}\left(x_{i}\right)$ are, based on Bellman and Zadeh [22], expanded to the fuzzy decision $D$, which adjusts the relative weights of the purposes and results of each using weights. In this case,

$\mu_{D}\left(z_{m}\right)=u_{A} \cdot \mu_{A}\left(z_{m}\right)+u_{B} \cdot \mu_{B}\left(z_{m}\right)$.

However, because

$u_{A}+u_{B}=1$

is obtained, it is defined as

$\boldsymbol{u}_{A}=\frac{w_{A}}{\left(w_{A}+w_{B}\right)}$,

$\boldsymbol{u}_{B}=\frac{w_{B}}{\left(w_{A}+w_{B}\right)}$.

The average characteristic value $\boldsymbol{L}_{\boldsymbol{F} 3 \boldsymbol{W}}$ in the weighted three-factor information channel model is defined as follows:

$$
\begin{aligned}
& \boldsymbol{L}_{F 3 W} \\
& =\sum_{m=1}^{n}\left(\frac{\boldsymbol{c}_{m}^{v_{C}}}{\left\{\boldsymbol{u}_{A} \cdot \boldsymbol{\mu}_{A}\left(\boldsymbol{z}_{m}\right)+\boldsymbol{u}_{B} \cdot \boldsymbol{\mu}_{B}\left(\boldsymbol{z}_{m}\right)\right\}^{v_{A B}}}\right) \cdot \boldsymbol{r}_{m}
\end{aligned}
$$

Because $\boldsymbol{c}_{\boldsymbol{m}}$ and $\mathbf{1} / \boldsymbol{\mu}_{\boldsymbol{D}}\left(\boldsymbol{z}_{\boldsymbol{m}}\right)$ in $\boldsymbol{L}_{\boldsymbol{F} 3 \boldsymbol{W}}$ in Equation (24) become a multiplication model (the form of the product), the weights $\left(\boldsymbol{v}_{A B}\right.$ and $\left.v_{C}\right)$ on the denominator $\boldsymbol{c}_{\boldsymbol{m}}$ and the numerator $\left\{\boldsymbol{u}_{A} \times \boldsymbol{\mu}_{A}\left(\boldsymbol{z}_{\boldsymbol{m}}\right)+\boldsymbol{u}_{B} \times \boldsymbol{\mu}_{B}\left(\boldsymbol{z}_{\boldsymbol{m}}\right)\right\}$ of the average characteristic value $\boldsymbol{L}_{F 3 W}$ are represented by powers. Hence, because $\boldsymbol{v}_{A B}+\boldsymbol{v}_{C}=\mathbf{1}$, these are defined as follows:

$$
\begin{aligned}
& v_{A B}=1-\frac{w_{C}}{\left(w_{A}+w_{B}+w_{C}\right)}, \\
& v_{C}=\frac{w_{C}}{\left(w_{A}+w_{B}+w_{C}\right)} .
\end{aligned}
$$

Hence, the randomness-fuzziness entropy $\left(\boldsymbol{H}_{\boldsymbol{R} F 3 \boldsymbol{W}}\right)$ in the weighted three-factor information channel model is formulated using Equation (27).

$$
\begin{aligned}
H_{R F 3 W}= & -\sum_{m=1}^{n} r_{m} \cdot \log r_{m} \\
& +\sum_{m=1}^{n} r_{m} \cdot G_{2 m}
\end{aligned}
$$

where $\boldsymbol{G}_{2 m}$ is the entropy regarding fuzziness and is defined by Equation (28):

$$
\begin{aligned}
& \boldsymbol{G}_{2 m}=-\left\{\boldsymbol{u}_{A} \cdot \boldsymbol{\mu}_{A}\left(\mathbf{z}_{m}\right)+\boldsymbol{u}_{B} \cdot \boldsymbol{\mu}_{B}\left(\boldsymbol{z}_{m}\right)\right\} \\
& \cdot \log \left\{\boldsymbol{u}_{A} \cdot \mu_{A}\left(z_{m}\right)+u_{B} \cdot \mu_{B}\left(z_{m}\right)\right\} \\
& -\left[\mathbf{1}-\left\{\boldsymbol{u}_{A} \cdot \boldsymbol{\mu}_{A}\left(\boldsymbol{z}_{\boldsymbol{m}}\right)+\boldsymbol{u}_{B} \cdot \boldsymbol{\mu}_{B}\left(\boldsymbol{z}_{\boldsymbol{m}}\right)\right\}\right] \\
& \cdot \log \left[1-\left\{u_{A} \cdot \mu_{A}\left(z_{m}\right)+u_{B} \cdot \mu_{B}\left(z_{m}\right)\right\}\right] \text {. }
\end{aligned}
$$

The problem of estimating selection ratio $\boldsymbol{r}_{\boldsymbol{m}}$ is represented by the following equation:

$$
\begin{aligned}
& R_{3 W}=\frac{H_{R F 3 W}}{L_{F 3 W}}-\lambda \cdot\left(\sum_{m=1}^{n} r_{m}-1\right) \\
& \rightarrow \max .
\end{aligned}
$$

As Equation (29) is convex with respect to $\boldsymbol{r}_{\boldsymbol{m}}$, the following equation can be solved for model 2 by partially differentiating Equation (29) with respect to $\boldsymbol{r}_{\boldsymbol{m}}$ and setting the differential to 0 :

$$
\begin{aligned}
& \boldsymbol{r}_{m} \exp \left[\left(\frac{G_{2 m}-c_{m}^{v_{C}}}{\left\{\boldsymbol{u}_{A} \cdot \mu_{A}\left(z_{m}\right)+u_{B} \cdot \mu_{B}\left(z_{m}\right)\right\}^{v_{A B}}}\right)\right] \\
& \cdot \frac{\boldsymbol{H}_{R F 3 W}}{L_{F 3 W}} \\
& =\exp \left[G_{2 m}\right] \cdot W_{2}^{\frac{c_{m}^{v_{C}}}{\left\{u_{A} \cdot \mu_{A}\left(z_{m}\right)+u_{B} \cdot \mu_{B}\left(z_{m}\right)\right\}^{v_{A B} B}}}
\end{aligned}
$$


where $\boldsymbol{W}_{2}=\boldsymbol{e} \boldsymbol{x p}\left[-\frac{\boldsymbol{H}_{R F 3 W}}{\boldsymbol{L}_{F 3 W}}\right]$ and the sum of all $\boldsymbol{r}_{\boldsymbol{m}}$ values is 1 . Therefore, $\boldsymbol{W}_{\mathbf{2}}$ satisfies Equation (31):

$$
\sum_{m=1}^{n} \exp \left[G_{2 m}\right] \cdot W_{2}^{\frac{c_{m}^{v} C}{\left\{u_{A} \cdot \mu_{A}\left(z_{m}\right)+u_{B} \cdot \mu_{B}\left(z_{m}\right)\right\}^{v} A B}}=1
$$

\section{Application to supplier selection}

Today, when companies are required to pursue a competitive advantage for their entire supply chain through alliances and collaborations with other companies, optimization within just one individual firm is insufficient. Therefore, it is necessary to achieve optimization for the entire group of companies that constitute the supply chain. Moreover, it is important to select suppliers that will function as partners to optimize the entire group of companies in the supply chain. However, when selecting a supplier, the DM must not only use clear, quantitative information to evaluate the supplier but also qualitative information in uncertain forms and with insufficient supporting evidence, making these decisions difficult.

\subsection{Supplier selection problem}

In recent years, the evaluation of supplier performance and selection of the optimum supplier have become the most important purchasing management activities for achieving overall supply chain management optimization. However, a DM selecting suppliers must do so while considering multiple selection criteria; hence, this is an MCDM problem [23, 24].

Because the supplier selection criteria consist not only of quantitative information but also considerable amounts of qualitative, vague information; to address this problem, methods of evaluating suppliers based on a fuzzy-theory approach have been proposed $[2,25]$. Ho, $\mathrm{Xu}$, and Dey [26] classified MCDM approaches for supplier evaluation and selection into independent approaches and integrated approaches. Furthermore, they categorized integrated approaches into three categories: integrated AHP approaches, integrated fuzzy approaches, and other approaches. Moreover, they classified integrated fuzzy approaches into six categories as follows: (1) integrated fuzzy and AHP, (2) integrated fuzzy, AHP, and cluster analysis, (3) integrated fuzzy genetic algorithm, (4) integrated fuzzy and multiobjective programming, (5) integrated fuzzy and quality function deployment, and (6) integrated fuzzy and simple multiattribute rating technique (SMART).

Amid et al. [27] considered supplier selection to be a multiobjective decision-making problem and paid attention to the need to attach DM weights to each of the selection criteria. As the vagueness of information makes decision making difficult, they proposed a fuzzy multiobjective model that reflected the DM's weights. $\mathrm{Ho}, \mathrm{Xu}$, and Dey [26] positioned the model of Amid et al. [27] as integrated fuzzy and multiobjective programming in integrated fuzzy approaches. They introduced nine integrated fuzzy approaches. Among the nine approaches, that of Amid et al. [27] has three criteria, and the DM's weights are set as given coefficients representing the three criteria. Therefore, to evaluate the performance of the three-factor fuzzy information channel model (model 1) and weighted three-factor fuzzy information channel model (model 2) of this study, we compared them with the model of Amid et al. [27].

Formulations of supplier selection problems with the fuzzy multiobjective model presented by Amid et al. [27] are summarized in the following steps:

Step 1: Construct the supplier selection model according to the criteria and constraints of the buyer and suppliers.

Step 2: Solve the multiobjective supplier selection problem as a single-objective supplier selection problem using only one objective each time. This value is the best value for this objective as other objectives are absent.

Step 3:From the results of step 2, determine the corresponding values for every objective in each solution derived.

Step 4:From step 3, for each objective function, find a lower bound and an upper bound corresponding to the set of solutions for each objective. Let $Z_{j}^{-}$and $Z_{j}^{+}$denote the lower bound and upper bound for the $j^{\text {th }}$ objective $\left(Z_{j}\right)$, respectively.

Step 5:For the objective functions and fuzzy constraints, find the membership function.

Step 6:From step 5 and the DM's preferences, based on fuzzy convex decision making, formulate the equivalent crisp model of the fuzzy optimization problem.

Step 7:Find the optimal solution vector $x^{*}$, which is an efficient solution of the original multiobjective supplier selection problem with the DM's preferences.

The model of Amid et al. [27] is a relatively simple mathematical model (fuzzy multiobjective linear model). In addition, in the method of Amid et al. [27], an FMCDM model was developed for the first time for the supplier selection problem, in which different weights can be considered for various objectives. Subsequently, the FMCDM methods have evolved into integrated models and hybrid models, and FMCDM methods are becoming more sophisticated and complicated. Therefore, we believe it would be meaningful to compare the simplicity and ease of calculation of the models proposed in this study with the model of Amid et al. [27], which is among the first (early) simple weighted FMCDM models.

In this research, we used the same numerical examples as in the study by Amid et al. [27] (p.401, Table1). Specifically, we consider three suppliers, $z_{1}, z_{2}$, and $z_{3}$ and three supplier selection criteria: cost, quality, and service. These three criteria are widely used as the top 
Table 1 Supplier information

\begin{tabular}{cccc}
\hline Supplier & Cost & $\begin{array}{c}\text { Quality (degree-of- } \\
\text { membership functions) }\end{array}$ & $\begin{array}{c}\text { Service (degree-of- } \\
\text { membership functions) }\end{array}$ \\
\hline Supplier $z_{1}$ & 3 & 0.85 & 0.75 \\
Supplier $z_{2}$ & 2 & 0.80 & 0.90 \\
Supplier $z_{3}$ & 5 & 0.95 & 0.85 \\
\hline
\end{tabular}

Table 2 List of suppliers' $\boldsymbol{\mu}_{D}\left(z_{m}\right)$

\begin{tabular}{cc}
\hline Supplier & $\mu_{D}\left(z_{m}\right)$ \\
\hline Supplier $z_{1}$ & $\frac{0.35}{0.35+0.25} \times 0.85+\frac{0.25}{0.35+0.25} \times 0.75=0.808$ \\
Supplier $z_{2}$ & $\frac{0.35}{0.35+0.25} \times 0.80+\frac{0.25}{0.35+0.25} \times 0.90=0.842$ \\
Supplier $z_{3}$ & $\frac{0.35}{0.35+0.25} \times 0.95+\frac{0.25}{0.35+0.25} \times 0.85=0.908$ \\
\hline
\end{tabular}

Table 3 Selection ratios for each supplier (model 1, model 2, and prior research [27])

\begin{tabular}{ccccc}
\hline Supplier & Model 1 & Model 2 & $\begin{array}{c}\text { Prior research fuzzy multi- } \\
\text { objective linear model [27] }\end{array}$ & $\begin{array}{c}\text { Prior research fuzzy multi-objective } \\
\text { linear model } \alpha \text {-cut approach [27] }\end{array}$ \\
\hline Supplier $z_{1}$ & 0.314 & 0.337 & 0.084 & 0.334 \\
Supplier $z_{2}$ & 0.563 & 0.396 & 0.378 & 0.381 \\
Supplier $z_{3}$ & 0.122 & 0.267 & 0.538 & 0.285 \\
\hline
\end{tabular}

three criteria that affect supplier selection [27-33]. However, in this research cost was considered quantitative and crisp information, while quality and service while quality and service information were unclear characteristic values (fuzzy information). These were ascertained as degree-of-membership functions (Table 1). Specifically, $85 \%, 80 \%$, and $95 \%$, which are the quality values for suppliers $z_{1}, z_{2}$, and $z_{3}$, respectively, were considered to be degree-ofmembership functions $\mu_{A}\left(z_{1}\right)=0.85, \mu_{A}\left(z_{2}\right)=0.80$, and $\mu_{A}\left(z_{3}\right)=0.95$, and suppliers' service values of $75 \%$, $90 \%$, and $85 \%$ were considered to be degree-ofmembership functions $\mu_{B}\left(z_{1}\right)=0.75, \mu_{B}\left(z_{2}\right)=0.90$, and $\mu_{B}\left(z_{3}\right)=0.85$, respectively.

Additionally, the DM's subjective weights for cost, quality, and service, $w_{C}, w_{A}$, and $w_{B}$, were set as 0.2 , 0.35 , and 0.25, respectively, as in Amid et al. [27] (p.402) Table 2 lists $\mu_{D}\left(z_{m}\right)$ values calculated using Equation (21) for each supplier.

Furthermore, $u_{A}$ and $u_{B}$, calculated using Equations (22) and Equation (23), respectively, are as follows:

$u_{A}=\frac{w_{A}}{\left(w_{A}+w_{B}\right)}=\frac{0.35}{0.35+0.25}=0.583$,

$u_{B}=\frac{w_{B}}{\left(w_{A}+w_{B}\right)}=\frac{0.25}{0.35+0.25}=0.417$.

In addition, $v_{A B}$ and $v_{C}$, calculated using Equations (24) and Equation (25), respectively, are as follows:

$$
\begin{aligned}
& v_{A B}=1-\frac{w_{C}}{\left(w_{A}+w_{B}+w_{C}\right)}=1-\frac{0.2}{(0.35+0.25+0.20)}=\frac{3}{4}, \\
& v_{C}=\frac{w_{C}}{\left(w_{A}+w_{B}+w_{C}\right)}=\frac{0.2}{(0.35+0.25+0.20)}=\frac{1}{4} .
\end{aligned}
$$

\subsection{Comparison of models 1 and 2}

The results of application of the models to the sample problem are listed in Table 3. For model 1, $r_{1}=0.314$, $r_{2}=0.563$, and $r_{3}=0.122$, while for model $2, r_{1}=$ $0.314, r_{2}=0.563$, and $r_{3}=0.122$ (corresponding to suppliers $z_{1}, z_{2}$, and $z_{3}$, respectively). In these two models, we used the cost, which is quantitative and crisp information, and quality and service, which are qualitative and ambiguous information, and considered the criteria of both qualitative and quantitative information. We verified that these models could easily obtain the DMs' selection ratios. They could estimate the hierarchical effects of various criteria other than cost, quality, and service considered in the above application example using a very simple model of entropy or fuzzy entropy maximization. Furthermore, our models are simple, and the DMs' selection ratios can be easily obtained by the convergence calculation (goal seek) of spreadsheet software.

In model 1, where the DM's weights for each selection criterion were not considered, the $z_{2}$ selection ratio was the highest, followed in order by $z_{1}$ and $z_{3}$, underscoring actual cost values. However, in model 2 , in which weights were considered, the weight for quality, $w_{A}=0.35$, had a significant effect, and for $z_{2}$, the lowest quality membership degree was 0.80 . Therefore, compared to model 1 , the selection ratio decreased by 0.167 . This result is persuasive. On the other hand, for $z_{3}$, the cost was high, but the highest membership degree for quality was 0.95 . Therefore, compared to model 1 , the selection ratio increased by 0.145 , implying that the selection ratio became more than double. The decrease for the $z_{2}$ selection ratio practically covered the increase for the $z_{3}$ selection ratio. In this manner, for model 2, it was possible to reflect the DM's weights for the selection criteria.

\subsection{Comparison with other methods}

Table 3 also lists the results of the selection ratios obtained from the fuzzy multiobjective linear model used in prior research [27] and from the $\alpha$-cut approach 
of the fuzzy multiobjective linear model with the selection ratio obtained [27]. The optimum solutions obtained in prior research were $r_{1}=0.084, r_{2}=0.378$, and $r_{3}=0.538$ when the $\alpha$-cut level was 0.187 ; the solutions obtained through the $\alpha$-cut approach, which performs a simulation while changing the $\alpha$-cut level from 0.187 up to 0.654 in steps of 0.05 , were $r_{1}=0.334$, $r_{2}=0.381$, and $r_{3}=0.285$ when the $\alpha$-cut level was 0.437 . The values obtained using model 2 were close to the values obtained using the $\alpha$-cut approach in prior research.

The weighted three-factor fuzzy information channel model proposed here treats the cost as clear, crisp information and the other two selection criteria as qualitative, vague information. Therefore, it can obtain the single most suitable solution per the DM's preference through a simple numerical calculation.

\section{Summary and conclusions}

We expanded the two-factor fuzzy information channel model for MCDM problems to propose a three-factor fuzzy information channel model. Moreover, we successfully expanded this model to a weighted threefactor fuzzy information channel model by reflecting the evaluation weight in the DM's value judgments. These models express the decision-making process when DMs decide the selection ratio based on subjective judgments mediated by vagueness related to randomness and vagueness related to fuzziness; therefore, they resolve MCDM problems based on randomness-fuzziness entropy by using both entropy related to randomness and entropy related to fuzziness.

Furthermore, we used a numerical example to compare supplier selection ratios obtained with the weighted three-factor fuzzy information channel model (model 2) to those obtained using a fuzzy multiobjective linear model from prior research. Consequently, we showed that model 2 yields practically the same supplier selection ratios as those obtained from the $\alpha$-cut approach using the fuzzy multiobjective linear model. Model 2, which reflects the weights of the DM's criteria, duplicates the human decision-making process in a concise form, permitting the estimation of the supplier selection ratio best suited to the DM's preferences. We verified that it could acquire the solution in a simpler manner compared with the fuzzy multiobjective linear model. As described in 5.2, by using our application example, we could confirm and prove our model's features and advantages, (a) to (d) listed in section 1.

Models 1 and 2 proposed here are both three-factor models that use one type of quantitative information and two types of qualitative fuzzy information; however, in future, we intend to expand the application range of these models by building a model expanded to four factors spanning two types of quantitative information. Another challenge for the future is the development of a multifactor fuzzy information channel model expanded to many factors.

\section{Appendix A}

As Equation (3) is convex with respect to $p_{i}$, when Equation (3) is partially differentiated with respect to $p_{i}$ and the differential is set to 0 , the selection ratio (selection probability) $p_{i}$ is represented as follows:

$p_{i}=w^{-x_{i}}$

where $w=e^{E / L}$.

Here, because the sum of the selection ratios is 1 as in Equation (A.2), in Equation (A.1), by numerically obtaining $w$ so that the sum of $p_{i}$ becomes 1 and by substituting the value of $w$ into Equation (A.1), the selection ratio $p_{i}$ that satisfies Equation (3) can be obtained [8]:

$$
\sum_{i=1}^{n} p_{i}=\sum_{i=1}^{n} w^{-x_{i}}=1
$$

\section{Appendix B}

As Equation (9) is convex with respect to $q_{j}$, when Equation (9) is partially differentiated with respect to $q_{j}$ with the differential set to 0, Equation (B.1) is obtained, which can be converted to Equation (B.2) [20]:

$$
\begin{aligned}
& \frac{\partial R_{1}}{\partial q_{j}}=\frac{H_{j}^{\prime} L_{F 1}-H_{j} L_{F 1}^{\prime}}{\left(L_{F 1}\right)^{2}}-\lambda=0 \\
& =\frac{\left(-\log q_{j}-1+\left[-\mu_{j} \cdot \log \mu_{j}-\left(1-\mu_{j}\right) \cdot \log \left(1-\mu_{j}\right)\right]\right) \cdot L_{F 1}-E_{R F 1} \cdot \mu_{j}}{\left(L_{F 1}\right)^{2}} \\
& -\lambda=0
\end{aligned}
$$

where $H_{j}^{\prime}$ and $L_{j 1}^{\prime}$ in Equation (B.1) are $H_{j}^{\prime}=\frac{\partial H_{j}}{\partial q_{j}}$ and $L_{j 1}^{\prime}=\frac{\partial L_{F 1}}{\partial q_{j}}$, respectively.

When both sides of Equation (B.2) are multiplied by $q_{j}$ and the sum over $j$, where $j=1$ to $n$, is taken, Equation (B.3) is obtained. Rearranging Equation (B.3) yields Equation (B. 4):

$$
\begin{aligned}
& \frac{\left[-\sum_{j=1}^{n} q_{j} \cdot \log q_{j}-\sum_{j=1}^{n} q_{j}+\sum_{j=1}^{n} q_{j} \cdot\left\{-\mu_{j} \cdot \log \mu_{j}-\left(1-\mu_{j}\right) \cdot \log \left(1-\mu_{j}\right)\right\}\right] \cdot L_{F_{1}}-E_{R F 1} \cdot \sum_{j=1}^{n} q_{j} \cdot \mu_{j}}{\left(L_{F 1}\right)^{2}} \\
& \frac{-\lambda \cdot \sum_{j=1}^{n} q_{j}=0}{\left(E_{R F 1}-1\right) \cdot L_{F 1}-E_{P F 1} \cdot L_{F 1}}-\lambda=0 .
\end{aligned}
$$

When Equation (B. 4) is rearranged to solve for $\lambda$, it becomes Equation (B. 5) [20]: 
$\lambda=-\frac{1}{L_{F 1}}$

Here, to eliminate $\lambda$ in Equation (B.5), when $\lambda$ in Equation (B.5) is substituted into Equation (B.2), it becomes Equation (B. 6):

$$
\begin{gathered}
\frac{\left[-\log q_{j}+\left\{-\mu_{j} \cdot \log \mu_{j}-\left(1-\mu_{j}\right) \cdot \log \left(1-\mu_{j}\right)\right\}\right] \cdot L_{F 1}-E_{R F 1} \cdot \mu_{j}-(-1) \cdot L_{F 1}}{\left(L_{F 1}\right)^{2}} \\
=0 .
\end{gathered}
$$

Then, to obtain $q_{j}$, Equation (B. 6) is arranged to solve for $\log q_{j}$, resulting in Equation (B. 7):

$$
\begin{gathered}
\log q_{j}=\left\{-\mu_{j} \cdot \log \mu_{j}-\left(1-\mu_{j}\right) \cdot \log \left(1-\mu_{j}\right)\right\} \\
-\frac{E_{R F 1} \cdot \mu_{j}}{L_{F 1}} .
\end{gathered}
$$

Further, when the left-hand side of Equation (B. 7) is converted in terms of $q_{j}$, Equation (B.8) is obtained:

$q_{j}=e^{\left\{-\mu_{j} \cdot \log \mu_{j}-\left(1-\mu_{j}\right) \cdot \log \left(1-\mu_{j}\right)\right\}} \cdot w^{-\mu_{j}}$,

where $w=e^{\frac{E_{R F 1}}{L_{F 1}}}$.

Therefore, by obtaining $w$ numerically so that the sum of $q_{j}$ in Equation (B.8) becomes 1 and by substituting the value of $w$ into Equation (B.8), it is possible to estimate selection probability $q_{j}[20]$.

\section{Appendix C}

As Equation (13) is convex with respect to $q_{j}$, when Equation (13) is partially differentiated with respect to $q_{j}$ and the differential is set to 0 , the solution to Equation (C.1) is obtained:

$$
\begin{aligned}
q_{j} & =\exp \left[\left(\frac{G_{j}-t_{j}}{\mu_{A}\left(P_{j}\right)}\right)\right] \cdot \frac{H_{R F 2}}{L_{F 2}} \\
& =\exp \left[G_{j}\right] \cdot W^{\overline{t_{j}\left(P_{j}\right)}}
\end{aligned}
$$

where $W=\exp \left[-\frac{H_{R F 2}}{L_{F 2}}\right]$ and the sum of $q_{j}$ values is 1. Therefore, $W$ satisfies Equation (C.2) [21]:

$$
\sum_{j=1}^{n} \exp \left[G_{j}\right] \cdot W^{\frac{t_{j}}{\mu_{A}\left(P_{j}\right)}}=1
$$

\section{Acknowledgments}

We would like to thank Editage (www.editage.com) for English language editing and Publication Support.

\section{References}

[1] E.K. Zavadskas, Z. Turskis: Multiple criteria decision making (MCDM) methods in economics: An overview. Technological and Economic Development of Economy, 2 (2011), 397-427.

[2] J.J.H. Liou, G.H. Tzeng: Comments on "multiple criteria decision making (MCDM) methods in economics: an overview". Technological and Economic Development of Economy, 18(4), (2012), 672-695.

[3] E.K. Zavadskas, Z. Turskis, S. Kildiene: State of art surveys of overviews on MCDM/MADM methods. Technological and Economic Development of Economy, 20(1), (2014), 165-179.

[4] C. Kahraman, S.C. Onar, B. Oztaysi: Fuzzy multicriteria decision-making: A literature review. International Journal of Computational Intelligence Systems, 8(4), (2015), 637-666.

[5] E. Herrera-Viedma: Fuzzy sets and fuzzy logic in multi-criteria decision making. The 50th anniversary of Prof. Lotfi Zadeh's theory: introduction, Technological and Economic Development of Economy, 21(5), (2015), 677-683.

[6] A. Mardani, A. Jusoh, E.K. Zavadskas: Fuzzy multiple criteria decision-making techniques and applications - Two decades review from 1994 to 2014. Expert Systems with Applications, 42 (2015), 4126-4148.

[7] N. Honda: What is fuzzy entropy? Mathematical Sciences, "Entropy" Its many aspects, (Special Issue), (1992), 134-141. [in Japanese]

[8] K. Kunisawa: Entropy model. Union of Japanese Scientists and Engineers, (1975), 36-46. [in Japanese]

[9] L.A. Zadeh: Fuzzy sets. Information and Control, 8 (1965), 338-353.

[10]A. De Luca, S. Termini: A definition of a nonprobabilistic entropy in the setting of fuzzy sets theory. Information and Control, 20(4), (1972), 301312.

[11]R.R. Yager: On the measure of fuzziness and negation Part I : Membership in the unit interval. International Journal of General Systems, 5 (1979), 221-229.

[12] M. Collan, M. Fedrizzi, P. Luukka: New closeness coefficients for fuzzy similarity based fuzzy TOPSIS: An approach combining fuzzy entropy and multidistance. Advances in Fuzzy Systems, 2015(7), (2015), 1-12.

[13]I. Erol, S. Sencer, R. Sari: A new fuzzy multicriteria framework for measuring sustainability performance of a supply chain. Ecological Economics, 70 (2011), 1088-1100. 
[14]H. Garg, N. Agarwal, A. Choubey: Entropy based multi-criteria decision making method under fuzzy environment and unknown attribute weights. Global Journal of Technology and Optimization, 6(3), (2015), 1-4.

[15] D. Joshi, S. Kumar: Intuitionistic fuzzy entropy and distance measure based TOPSIS method for multicriteria decision making. Egyptian Informatics Journal, 15(2014), 97-104.

[16] S. Lee, T.O. Ting: Uncertainty evaluation via fuzzy entropy for multiple facts. International Journal of Electronic Commerce Studies, 4(2), (2013), 313-322.

[17] C. Wei, Y. Zhang: Entropy measures for intervalvalued intuitionistic fuzzy sets and their application in group decision-making. Mathematical Problems in Engineering, 2015(2015), 1-13.

[18] H. Yamashita: Randomness and fuzziness of fuzzy event. Journal of Japan Association for Management Systems, 12(2), (1995), 41-46. [in Japanese]

[19] T. Nishikawa, M. Imai, Y. Nakagawa: Application of fuzzy theory to human information processing. In Proceedings of the Third European Workshop on Fuzzy Decision Analysis and Neural Networks for Management, Planning and Optimization, Dortmund, Germany, (1998), 91-97.

[20]H. Yamashita: On the role of entropy in entropy models. The Bulletin of the Faculty of Commerce, Meiji University, 84(2), (2002), 71-88. [in Japanese].

[21] H. Yamashita: A double factor model for fuzzy information channel and the labeling effects of foods. The Bulletin of the Faculty of Commerce, Meiji University, 92(1), (2010), 1-10. [in Japanese]

[22]R.E. Bellman, L.A. Zadeh: Decision-making in a fuzzy environment. Management Science, 17(4), (1970), B141-164.

[23] G.W. Dickson GW: An analysis of vendor selection systems and decisions. Journal of Purchasing \& Supply Management, 2(1), (1966), 5-17.
[24] C.A. Weber, J.R. Current, W.C. Benton: Vendor selection criteria and methods. European Journal of Operational Research, 50(1991), 2-18.

[25] C.C. Li, Y.P. Fun, J.S. Hung: A new measure for supplier performance evaluation. IIE Transactions, 29 (1997), 753-758.

[26] W. Ho, X. Xu, P.K. Dey: Multi-criteria decision making approaches for supplier evaluation and selection: A literature review. European Journal of Operational Research, 202 (2010), 16-24.

[27] A. Amid, S.H. Ghodsypour, C. O’Brien: Fuzzy multiobjective linear model for supplier selection in a supply chain. International Journal of Production Economics, 104(2), (2006), 394-407.

[28] A. Amid, S.H. Ghodsypour, C. O’Brien: A weighted additive fuzzy multi-objective model for the supplier selection problem under price breaks in a supply chain, International Journal of Production Economics, 121(2), (2009), 323-332.

[29] A. Amid, S.H. Ghodsypour, C. O'Brien: A weighted max-min model for fuzzy multi-objective supplier selection in a supply chain. International Journal of Production Economics, 131(1), (2011), 139-145.

[30] O. Jadidi, S. Zolfaghari, S. Cavalieri: A new normalized goal programming model for multiobjective problems: A case of supplier selection and order allocation. International Journal of Production Economics, 148(2014), 158-165.

[31]M. Kumar, P. Vart, R. Shankar: A fuzzy goal programming approach for supplier selection problem in a supply chain. Computers \& Industrial Engineering, 46(1), (2004), 69-85.

[32] M. Kumar, P. Vart, R. Shankar: A fuzzy programming approach for vendor selection problem in a supply chain. International Journal of Production Economics, 101(2), (2006), 273-285.

[33] V. Wadhwa, A.R. Ravindran: Vendor selection in outsourcing. Computers \& Operations Research, 34(12), (2007), 3725-3737. 